\title{
Eyelash inversion in epiblepharon: Is it caused by redundant skin?
}

\author{
Hirohiko Kakizaki' \\ Igal Leibovitch ${ }^{2}$ \\ Yasuhiro Takahashi ${ }^{3}$ \\ Dinesh Selva ${ }^{4}$
}

'Department of Ophthalmology, Aichi Medical University, Nagakute, Aichi 480-I I95, Japan; ${ }^{2}$ Division of Oculoplastic and Orbital Surgery, Department of Ophthalmology, Tel-Aviv Medical Center,

Tel-Aviv University, Tel-Aviv, Israel; ${ }^{3}$ Department of Ophthalmology and Visual Sciences, Osaka City University Graduate School of Medicine, Osaka 545-8585, Japan; ${ }^{4}$ South Australian Institute of Ophthalmology and Discipline of Ophthalmology and Visual Sciences, University of Adelaide, Australia

Correspondence: Hirohiko Kakizaki Department of Ophthalmology, Aichi Medical University, Nagakute, Aichi 480-I 195, Japan

Tel +8156162331 I (Ext. 218I)

Fax +8I 561637255

Email cosme@dI.dion.ne.jp
Purpose: To evaluate the effect of redundant lower eyelid skin on the eyelash direction in epiblepharon.

Materials and methods: Asian patients with epiblepharon participated in this study. The lower eyelid skin was pulled downward in the upright position with the extent just to detach from eyelash roots, and the direction of the eyelashes was examined. These evaluations were repeated before surgery while the patients were lying supine under general anesthesia.

Results: The study included 41 lower eyelids of 25 patients ( 17 females, 8 males, average age; 5.6 years, 16 cases bilateral, 9 unilateral). In the upright position, without downward traction of the skin, the eyelashes were vertically positioned and touching the cornea. The redundant skin touched only the eyelash roots and had minimal contribution to eyelash inversion. With downward skin traction, there was no significant change in the eyelash direction. In the spine position, the eyelashes were touching the cornea, and there was marked redundant skin that was pushing the eyelashes inward. With downward skin traction, there was no significant change. Conclusions: The direction of lower eyelashes in patients with epiblepharon was less influenced by lower eyelid skin redundancy than previously considered. The redundant skin is only a possible aggravating factor to epiblepharon.

Keywords: epiblepharon, skin redundancy, upright, eyelash

\section{Introduction}

Epiblepharon is generally described as a condition in which a horizontal fold of redundant skin and the underlying pretarsal orbicularis oculi muscle tilt the eyelashes and push them against the globe. ${ }^{1}$ The tarsal plate, however, is not tilted and remains in its normal position. ${ }^{1,2}$

There are several possible etiologic factors that could be involved in the pathogenesis of epiblepharon. ${ }^{3}$ The eyelid retractors may fail to gain access to skin and there may also be failure of interdigitation of septae in the subcutaneous plane. ${ }^{3}$ Another possible cause is a weak attachment of the pretarsal orbicularis oculi muscle and skin to the tarsus below, thus raising a skin fold near the eyelid margin and pushing the eyelashes toward the cornea. ${ }^{2,4}$ Hypertrophy of the orbicularis oculi muscle was also raised and is a possible etiologic factor. ${ }^{2,4}$

From our clinical impression, the eyelashes in the involved section of epiblepharon show a vertical direction irrespective of the extent of skin redundancy. We therefore examined the direct effect of the redundant skin on the direction of the eyelashes in a series of patients with epiblepharon.

\section{Methods}

The study included all patients of Asian descent who were diagnosed with epiblepharon and significant ocular irritation, with or without keratitis, and were scheduled for surgical correction under general anesthesia between August 2004 and October 2008. 
The study included 41 lower eyelids of 25 patients (17 females, 8 males, average age; 5.6 years, age range: $2-10$ years). Sixteen cases were bilateral and nine were unilateral (right side, 6; left side, 3). Patients gave their informed consent to the content of this study following an explanation.

Eyelash direction was examined while the patients were awake and in the upright position. The lower eyelid skin was then pulled downward to detach from eyelash roots and the direction of the lashes re-examined. The extent of force to pull the skin downward was gentle enough just to remove the effect of the skin pushing the eyelash roots. These evaluations were repeated before surgery while the patients were lying supine under general anesthesia. All examinations were performed by one of the authors (HK).

\section{Results}

We found that in the upright position, without downward traction of the skin, the eyelashes were vertically positioned and touching the cornea. The redundant skin touched only the eyelash roots and seemed to have a small contribution to eyelash inversion (Figure 1A). With downward skin traction, we noted no significant change in the direction of the eyelashes (Figure 1B).

In the supine position, under general anesthesia, the eyelashes were touching the cornea as well and there was marked redundant skin that was pushing the eyelashes inward (Figure 2A). With downward skin traction, there was no significant change in the direction of the eyelashes (Figure 2B).

\section{Discussion}

In our series of patients with epiblepharon, we found that the eyelashes were always in an upright direction, irrespective of the patients' position or manual downward traction of the skin (Figure 3).

In the supine position, however, eyelash inversion was aggravated. Although this could result from the effect of gravitational forces on the eyelashes, it could also be caused by an upward move of the lower eyelid skin. Therefore, the lower eyelid redundant skin should be regarded merely as a possible aggravating factor, rather than the main etiologic cause.

A contributing factor to the pathogenesis of epiblepharon is failure of the eyelid retractors to gain access to skin., ${ }^{2,3}$ Evidence to support this theory include our findings that downward skin traction did not change the direction of eyelashes, and the fact that epiblepharon tends to improve with age and facial bone maturation, ${ }^{2,4,5}$ which may tense the lower eyelid retractors, causing spontaneous improvement of the eyelash inversion.

The lower eyelid retractors are a double (anterior and posterior) layer structure. ${ }^{6}$ The anterior layer originates from the Lockwood's ligament, merges with the orbital septum and submuscular fibrous tissue and attaches to the anterior surface of the lower tarsal plate and subcutaneous layer through the orbicularis oculi muscle. The posterior layer is the main traction layer of the lower eyelid retractors, including the smooth muscle fibers, and inserts onto the anterior, inferior and posterior surfaces of the lower tarsal plate. As the tarsal plate does not roll inward but remains in its normal position in epiblepharon, ${ }^{1,2}$ the posterior layer probably has no significant role in the pathogenesis of this entity. We therefore believe that the main factor is the anterior layer of the lower eyelid retractors, and hence surgical techniques to reinforce the anterior layer, such as the Hotz procedure ${ }^{7}$

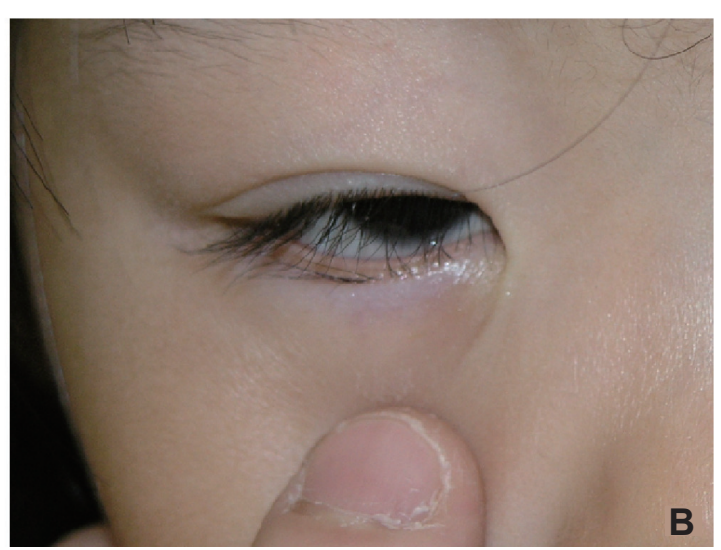

Figure I A) A 3-year-old girl. Upright position without downward traction of the skin. The eyelashes are directed vertically and rub on the cornea. The redundant skin only touches the eyelash roots and therefore it seems to have a minimal contributing role in eyelash inversion. B) Upright position with downward traction of the skin. The direction of the eyelashes is not changed significantly, and they are still directed to the globe. 

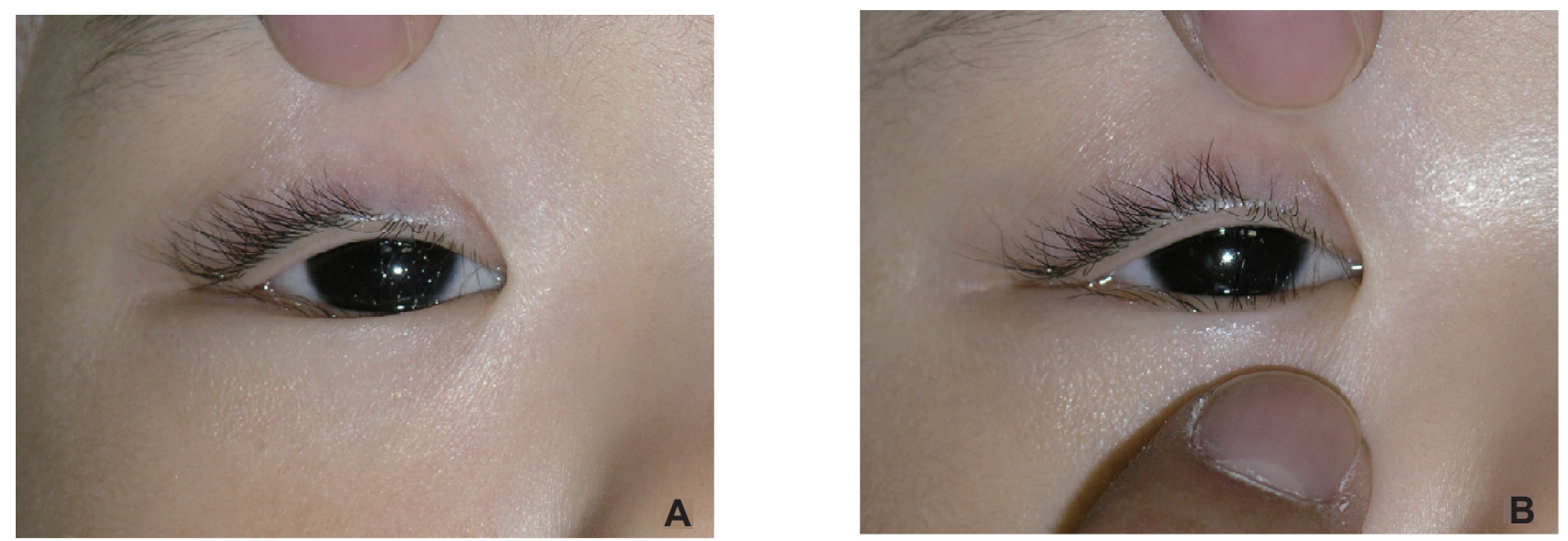

Figure 2 A) The same 3-year-old girl. Spine position during general anesthesia without downward traction of the skin. The eyelashes are directed toward the cornea. More redundant skin is observed in the lower eyelid than in the upright position (Figure IA) and seems to have more influence of eyelash direction. B) Spine position during general anesthesia with downward traction of the skin. The direction of the eyelashes has not changed significantly compared with the upright position (Figure IB).

or other anterior lamellar repositioning techniques, ${ }^{8}$ could provide the best surgical outcome.

An absent lower eyelid skin crease in patients with epiblepharon was considered to have supportive evidence that the lower eyelid retractor fibers fail to reach the skin. ${ }^{2,9}$ However, it should be remembered that most Asians have no lower eyelid skin crease, ${ }^{1,8}$ even if the anterior layer fibers of the lower eyelid retractors reach the skin. ${ }^{6}$ In addition, the fat in front of the lower eyelid retractors occupies the anterosuperior region of the lower eyelid, thus preventing the fibers from creating the lower eyelid skin crease.$^{10}$ It is therefore not recommended to create a surgical lower lid crease during epiblepharon surgery in Asians. ${ }^{1}$

A weak attachment of the pretarsal orbicularis muscle and skin to the underlying tarsus, that was raising a skin fold near the eyelid margin and pushing the eyelashes toward the cornea, was also considered as a possible etiology of epiblepharon. ${ }^{3-5}$ Our study found that redundant skin is only a possible aggravating factor and does not support this theory. When combining our findings and previous evidence that epiblepharon tends to improve with age, ${ }^{2,4,5}$ it seems that removing any redundant skin during surgery is

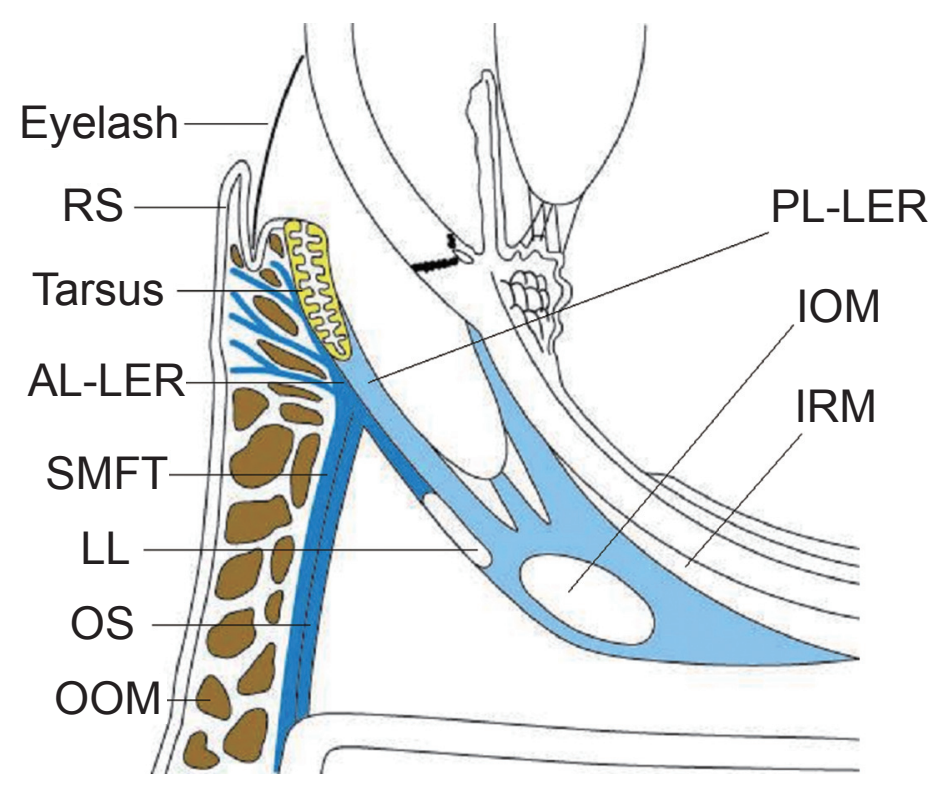

Figure 3 A diagram of epiblepharon. The eyelash is always directed upright, irrespective of skin redundancy.

Abbreviations: AL-LER, anterior layer of lower eyelid retractors; IOM, inferior oblique muscle; IRM, inferior rectus muscle; LL, Lockwood's ligament; OS, orbital septum; OOM, orbicularis oculi muscle; PL-LER, posterior layer of lower eyelid retractors; RS, redundant skin; SMFT, submuscular fibrous tissue. 
not essential, and that surgery should focus on changing the eyelash direction. Excision of skin helps little in improving the eyelash position and may prone to cause ectropion.

Hypertrophy of the orbicularis oculi muscle was also mentioned as a causative factor in epiblepharon, ${ }^{2-4}$ however, this is not supported by any microscopic studies. Interestingly, hypertrophy of the orbicularis oculi muscle was also believed to cause congenital entropion, ${ }^{2,3,5}$ but this theory was denied in the microscopic studies. ${ }^{11}$ Based on the "hypertrophy" theory, it was recommended to remove orbicularis muscle in epiblepharon surgery, ${ }^{1,8}$ but there is no evidence to justify debulking this muscle, unless it is required to allow effective rolling out of the eyelashes. ${ }^{8}$

In conclusion, we found that the direction of lower eyelashes in patients diagnosed with epiblepharon and significant ocular irritation, with or without keratitis, was less influenced by lower eyelid skin redundancy than previously considered. The redundant skin is only a possible aggravating factor to epiblepharon.

\section{Disclosure}

The authors report no financial support and no financial interest related to this manuscript.

\section{References}

1. Woo KI, Yi K, Kim YD. Surgical correction for lower lid epiblepharon in Asians. Br J Ophthalmol. 2000;84:1407-1410.

2. Jordan R. The lower-lid retractors in congenital entropion and epiblepharon. Ophthalmic Surg. 1993;24:494-496.

3. Preechawai P, Amrith S, Wong I, Sundar G. Refractive changes in epiblepharon. Am J Ophthalmol. 2007;143:835-839.

4. Johnson CC. Entropion. Am J Ophthalmol. 1968;66:1172-1175.

5. Levitt JM. Epiblepharon and congenital entropion. Am J Ophthalmol. 1957;44:112-113.

6. Kakizaki H, Zhao J, Nakano T, et al. The lower eyelid retractor consists of definite double layers. Ophthalmology. 2006;113:2346-2350.

7. Hotz FC. A new operation for entropion and trichiasis. Arch Ophthalmol. 1879;8:249-263.

8. Choo C. Correction of oriental epiblepharon by anterior lamellar reposition. Eye. 1996;10:545-547.

9. Millman AL, Mannor GE, Putterman AM. Lid crease and capsulopalpebral fascia repair in congenital entropion and epiblepharon. Ophthalmic Surg. 1994;25:162-165.

10. Carter SR, Seiff SR, Grant PE, Vigneron DB. The Asian lower eyelid: a comparative anatomic study using high-resolution magnetic resonance imaging. Ophthal Plast Reconstr Surg. 1998;14:227-234.

11. Tse DT, Anderson RL, Fratkin JD. Aponeurosis disinsertion in congenital entropion. Arch Ophthalmol. 1983;101:436-440. 